\title{
TERMINATING TABLEAUX FOR GRADED HYBRID LOGIC WITH GLOBAL MODALITIES AND ROLE HIERARCHIES
}

\author{
MARK KAMINSKI, SIGURD SCHNEIDER, AND GERT SMOLKA
}

Saarland University, Saarbrücken, Germany

e-mail address: \{kaminski,smolka\}@ps.uni-saarland.de

\begin{abstract}
We present a terminating tableau calculus for graded hybrid logic with global modalities, reflexivity, transitivity and role hierarchies. Termination of the system is achieved through pattern-based blocking. Previous approaches to related logics all rely on chain-based blocking. Besides being conceptually simple and suitable for efficient implementation, the pattern-based approach gives us a NExPTIME complexity bound for the decision procedure.
\end{abstract}

\section{INTRODUCTION}

Graded modal logic [12] is a powerful generalization of basic modal logic. Most prominently, graded modalities are used in description logics, rich modal languages tailored for knowledge representation that have a wide range of practical applications [3]. Graded modal logic allows to constrain the number of accessible states satisfying a certain property. So, the modal formula $\nabla_{n} p$ is true in a state $x$ if $x$ has at least $n+1$ successors satisfying $p$. Analogously to ordinary modal logic, graded modal logic can be extended by nominals [1]. The resulting language, graded hybrid logic, can be extended further by adding global modalities [13], which allow to specify properties that are to hold in all states.

Role hierarchies were first studied by Horrocks [16] in the context of description logics. Using inclusion assertions of the form $r \sqsubseteq r^{\prime}$, one can specify that the role (relation) $r$ is contained in the role $r^{\prime}$. Role hierarchies are of particular interest when considered together with transitivity assertions for roles [30, 4]. The description logic $\mathcal{S H O Q}$ [18] combines the expressive means provided by nominals, graded modalities, role hierarchies and transitive roles.

We present a terminating tableau calculus for graded multimodal logic extended by nominals, global modalities, reflexive and transitive roles, and role hierarchies. The modal language under consideration in the present work is equivalent to $\mathcal{S H O} \mathcal{Q}$ extended by reflexive roles and a universal role, both extensions also being known from $\mathcal{S} \mathcal{R O I} \mathcal{Q}$ [17].

1998 ACM Subject Classification: F.4.1, I.2.3, I.2.4.

Key words and phrases: Modal logic, hybrid logic, tableau calculi, decision procedures.

A preliminary version of this work appeared in 21.

네 
The most important difference of our approach to existing calculi for $\mathcal{S H O Q}$ and stronger logics [18, 19, 17] is the technique used to achieve termination of the tableau construction. The established tableau algorithms all rely on modifications of Kripke's chainbased blocking technique [27. Chain-based blocking assumes a precedence order on the nominals (also known as nodes or prefixes) of a tableau branch, and prevents processing of nominals that are subsumed by preceding nominals. In the simplest case, the precedence order is chosen to be the ancestor relation among nominals (ancestor blocking). In general, however, it may be any order that contains the ancestor relation (anywhere blocking [2, 28]). Ancestor blocking gives an exponential bound on the length of ancestor chains, resulting in a double exponential bound on the size of tableau branches. Depending on the choice of the precedence order, anywhere blocking can lower this bound to a single exponential. However, the size bound on tableau branches does not seem to translate easily to a complexity bound for the decision procedures in [18, 19, 17, ([18, 19] show a 2-NExPTime bound, while [17] leaves complexity open). We feel that the main difficulty in obtaining better complexity bounds is the algorithms being non-cumulative.

A tableau system is called cumulative if its rules never update or delete formulas. In contrast to most systems in the literature, calculi devised for description logics are often not cumulative. By giving up cumulativity, it is possible to obtain a more direct correspondence between tableau branches and the candidate models they represent. So, for instance, a noncumulative calculus may merge several nominals into one if the nominals are found to be semantically equivalent. In this way, one can achieve that every state of a candidate model is represented by exactly one nominal. This close correspondence is intuitive and may simplify model existence arguments. At the same time, non-cumulative rules are typically more complex than their cumulative counterparts, which may complicate the presentation of a calculus. More importantly, cumulative systems are usually more amenable to termination and complexity analysis. The problem with non-cumulative systems is that rules that can update or delete formulas may potentially undo earlier changes made to a tableau branch. For instance, consider two tableau branches $\Gamma$ and $\Delta$, where $\Delta$ is obtained from $\Gamma$ by some sequence of tableau rule applications. In a non-cumulative calculus, it is conceivable that by applying some rule to $\Delta$, we may obtain $\Gamma$ again. Clearly, such a calculus is non-terminating even if the size of tableau branches can be bounded. Often, termination of non-cumulative calculi can only be achieved if rule application follows some fixed strategy [5, 19, 17]. And even then, size bounds on tableau branches do not immediately yield time complexity bounds. To construct a branch of size $n$, a non-cumulative system may need significantly more than $n$ rule applications. Cumulative calculi, on the other hand, are guaranteed to enlarge the branch by at least one formula in every step. Therefore, a size bound on tableau branches can immediately be interpreted as an upper bound on the non-deterministic time complexity of the decision procedure.

Unlike [18, 19, 17, our calculus is cumulative. Cumulativity of the calculus in the presence of nominals is achieved following [24] by representing equality constraints via an equivalence relation on nominals. Termination of our system is achieved through patternbased blocking [23, 24]. Pattern-based blocking is conceptually simpler than chain-based techniques in that it does not need an order on the nominals, and seems promising as it comes to efficient implementation [15]. Pattern-based blocking provides an exponential bound on the size of tableau branches and on the number of tableau rule applications for a single branch. Thus it limits the complexity of the associated decision procedure to 
NExpTime. To deal with graded modalities, we extend the blocking conditions in [23, 24], preserving the exponential size bound on tableau branches.

It is worth noting that, despite of the close interplay between pattern-based blocking and abstract representation of state equality in the present work, the two techniques should be seen as independent and applicable in isolation from each other. In fact, pattern-based blocking was introduced in [23] for a non-cumulative system where equality was treated by means of a substitution operation on branches. Also, in previous work [24, we show how abstract treatment of equality can be combined with chain-based blocking to obtain cumulative, terminating tableau calculi for hybrid logic with converse modalities and the difference modality.

We begin by presenting a calculus for graded hybrid logic with global modalities. We argue that the blocking conditions used in [23, 24] are insufficient in the presence of graded modalities. We extend pattern-based blocking to account for the increased expressive power and argue the completeness and termination of the resulting calculus. In the second part of the paper, we extend our calculus further by allowing reflexivity, transitivity and inclusion assertions. It turns out that in the presence of inclusion assertions, the blocking condition used for the basic calculus needs to be extended once again.

\section{Graded Hybrid Logic with Global Modalities and Role Inclusion}

Following [22, 24], we represent modal logic in simple type theory (see, e.g., [10, 9]). This way we can make use of a rich syntactic and semantic framework and modal logic does not appear as an isolated formal system. We start with two base types B and S. The interpretation of $\mathrm{B}$ is fixed and consists of two truth values. The interpretation of $\mathrm{S}$ is a nonempty set whose elements are called worlds or states. Given two types $\sigma$ and $\tau$, the functional type $\sigma \tau$ is interpreted as the set of all total functions from the interpretation of $\sigma$ to the interpretation of $\tau$. We write $\sigma_{1} \sigma_{2} \sigma_{3}$ for $\sigma_{1}\left(\sigma_{2} \sigma_{3}\right)$.

We assume a countable set of names, which we partition into a countable set of variables and a set of constants. We employ three kinds of variables: Nominal variables $x, y, z$ of type $\mathrm{S}$, propositional variables $p, q$ of type $\mathrm{SB}$, and role variables $r$ of type $\mathrm{SSB}$. Nominal variables are called nominals for short, and role variables are called roles. We assume there are infinitely many nominals. We use the logical constants

$$
\perp, \top: \mathrm{B} \quad \neg: \mathrm{BB} \quad \vee, \wedge, \rightarrow: \mathrm{BBB} \quad \doteq: \mathrm{SSB} \quad \exists, \forall:(\mathrm{SB}) \mathrm{B}
$$

Terms are defined as usual. We write $s t$ for applications, $\lambda x . s$ for abstractions, and $s_{1} s_{2} s_{3}$ for $\left(s_{1} s_{2}\right) s_{3}$. We also use infix notation, e.g., $s \wedge t$ for $(\wedge) s t$.

Terms of type B are called formulas. We employ some common notational conventions: $\exists x . s$ for $\exists(\lambda x . s), \quad \forall x . s$ for $\forall(\lambda x . s)$, and $x \neq y$ for $\neg(x \doteq y)$. Given a set $X$ of nominals, we use the following abbreviation:

$$
D X:=\bigwedge_{\substack{x, y \in X \\ x \neq y}} x \neq y
$$

We use the following constants:

$$
\begin{aligned}
& \sqsubseteq:(\mathrm{SSB})(\mathrm{SSB}) \mathrm{B} \\
& R:(\mathrm{SSB}) \mathrm{B} \\
& T:(\mathrm{SSB}) \mathrm{B}
\end{aligned}
$$

$$
\begin{aligned}
r_{1} \sqsubseteq r_{2} & =\forall x y \cdot r_{1} x y \rightarrow r_{2} x y \\
R r & =\forall x \cdot r x x \\
T r & =\forall x y z \cdot r x y \wedge r y z \rightarrow r x z
\end{aligned}
$$


To the right of each constant is an equation defining its semantics. We call formulas of the form $r \sqsubseteq r^{\prime}$ (role) inclusion assertions. Formulas $R r$ and $T r$ are called reflexivity and transitivity assertions, respectively.

We write $\exists^{n} X$.s for $\exists x_{1} \ldots x_{n} . s$ if $|X|=n$ and $X=\left\{x_{1}, \ldots, x_{n}\right\}$. The modal constants are then defined as follows:

$$
\begin{aligned}
\dot{\neg} & :(\mathrm{SB}) \mathrm{SB} & \dot{\neg} p x & =\neg(p x) \\
\dot{\wedge} & :(\mathrm{SB})(\mathrm{SB}) \mathrm{SB} & (p \dot{\wedge} q) x & =p x \wedge q x \\
\dot{\vee} & :(\mathrm{SB})(\mathrm{SB}) \mathrm{SB} & (p \dot{\vee} q) x & =p x \vee q x \\
\left\langle \_\right\rangle_{n} & :(\mathrm{SSB})(\mathrm{SB}) \mathrm{SB} & \langle r\rangle_{n} p x & =\exists^{n+1} Y . D Y \wedge\left(\bigwedge_{y \in Y} r x y \wedge p y\right) \\
{[-]_{n} } & :(\mathrm{SSB})(\mathrm{SB}) \mathrm{SB} & {[r]_{n} p x } & =\forall^{n+1} Y .\left(\bigwedge_{y \in Y} r x y\right) \wedge D Y \rightarrow \bigvee_{y \in Y} p y \\
E_{n} & :(\mathrm{SB}) \mathrm{SB} & E_{n} p x & =\exists^{n+1} Y . D Y \wedge \bigwedge_{y \in Y} p y \\
A_{n} & :(\mathrm{SB}) \mathrm{SB} & A_{n} p x & =\forall^{n+1} Y . D Y \rightarrow \bigvee_{y \in Y} p y \\
- & : \mathrm{SSB} & \dot{x} y & =x \dot{=}
\end{aligned}
$$

where $n \geq 0$ in all equations

The semantics of boxes and diamonds is defined following [11, 31, 29]. Intuitively, it can be described as follows:

$E_{n} p:$ There are at least $n+1$ states satisfying $p$.

$A_{n} p$ : All states but possibly $n$ exceptions satisfy $p$.

$\langle r\rangle_{n} p$ : There are at least $n+1 r$-successors satisfying $p$.

$[r]_{n} p$ : All $r$-successors but possibly $n$ exceptions satisfy $p$.

In accordance with the usual modal intuition, "formulas" of modal logic are seen as predicates of type SB denoting sets of states. They can be represented as modal expressions according to the following grammar:

$$
t::=p|\dot{x}| \dot{\neg} t|t \dot{\wedge} t| t \dot{\vee} t\left|\langle r\rangle_{n} t\right|[r]_{n} t\left|E_{n} t\right| A_{n} t
$$

As with the propositional connectives, we use infix notation for $\dot{\lambda}$ and $\dot{V}$. Unlike with the propositional connectives, we assume the application of modal operators to have a higher precedence than regular functional application. So, for instance, we write $\dot{\neg}\langle r\rangle_{2} \dot{y} \dot{\vee} p x$ for $\left(\left(\dot{\neg}\left(\langle r\rangle_{2}(\dot{y})\right)\right) \dot{\vee} p\right) x$.

An interpretation is a function $\mathcal{I}$ mapping $\mathrm{B}$ to the set $\{0,1\}$, S to a non-empty set, a functional type $\sigma \tau$ to the set of all total functions from $\mathcal{I} \sigma$ to $\mathcal{I} \tau$, and every name $x: \sigma$ to an element of $\mathcal{I} \sigma$ (i.e., $\mathcal{I} x \in \mathcal{I} \sigma$ ) such that the logical constants get their usual meaning:

$$
\begin{aligned}
\mathcal{I} \perp=0 & \text { and } \mathcal{I} \top=1 & (\mathcal{I} \neg) a=1 & \Longleftrightarrow a=0 \\
(\mathcal{I} \wedge) a b=1 & \Longleftrightarrow a=1 \text { and } b=1 & (\mathcal{I} \vee) a b=1 & \Longleftrightarrow a=1 \text { or } b=1 \\
(\mathcal{I} \rightarrow) a b=1 & \Longleftrightarrow a=0 \text { or } b=1 & (\mathcal{I} \doteq) a b=1 & \Longleftrightarrow a=b \\
(\mathcal{I} \exists) f=1 & \Longleftrightarrow f a=1 \text { for some } a \in \mathcal{I S} & (\mathcal{I} \forall) f=1 & \Longleftrightarrow f a=1 \text { for all } a \in \mathcal{I} \mathrm{S}
\end{aligned}
$$

If $\mathcal{I}$ is an interpretation, $x: \sigma$ is a variable, and $a \in \mathcal{I} \sigma$, then $\mathcal{I}_{a}^{x}$ denotes the interpretation that agrees everywhere with $\mathcal{I}$ but possibly on $x$ where it yields $a$. Every interpretation $\mathcal{I}$ 
can be extended to a function $\hat{\mathcal{I}}$ that maps every term $s: \sigma$ to an element of $\mathcal{I} \sigma$ such that:

$$
\begin{aligned}
\hat{\mathcal{I}} x & =\mathcal{I} x \\
\hat{\mathcal{I}}(s t) & =(\hat{\mathcal{I}} s)(\hat{\mathcal{I}} t) \\
\hat{\mathcal{I}}(\lambda x . s) & =\left\{\left(a, \widehat{\mathcal{I}_{a}^{x}} s\right) \mid a \in \mathcal{I} \sigma\right\} \quad \text { if } x: \sigma
\end{aligned}
$$

Since $\hat{\mathcal{I}}$ is uniquely determined by $\mathcal{I}$, in the following we write $\mathcal{I} s$ for $\hat{\mathcal{I}} s$ for convenience. A modal interpretation is an interpretation that, in addition, satisfies the above equations defining the constants $\sqsubseteq, R, T, \dot{ᄀ}, \dot{\wedge}, \dot{V},\left\langle-_{-}\right\rangle_{n},[-]_{n}, E, A,-{ }_{-}$If $\mathcal{I} s=1$, we say that $\mathcal{I}$ satisfies $s$, or that $\mathcal{I}$ is a model of $s$. A modal interpretation $\mathcal{I}$ satisfies a set $\Gamma$ of formulas $(\mathcal{I}$ is a model of $\Gamma$ ) if $\mathcal{I}$ satisfies every formula in $\Gamma$. A formula (a set of formulas) is called satisfiable if it has a model.

\section{Graded Hybrid Logic with Global Modalities}

We begin with a tableau calculus for the restricted language without inclusion, reflexivity or transitivity assertions.

3.1. Branches. For the sake of simplicity, we define our tableau calculus on negation normal expressions, i.e., terms of the form:

$$
t::=p|\dot{\neg} p| \dot{x}|\dot{\neg} \dot{x}| t \dot{\wedge} t|t \dot{\vee} t|\langle r\rangle_{n} t\left|[r]_{n} t\right| E_{n} t \mid A_{n} t
$$

A branch $\Gamma$ is a finite set of formulas $s$ of the form

$$
s::=t x|r x y| x \doteq y|x \neq y| \perp
$$

where $t$ is a negation-normal modal expression of the above form. Formulas of the form $r x y$ are called accessibility formulas or edges. We use the formula $\perp$ to explicitly mark unsatisfiable branches. We call a branch $\Gamma$ closed if $\perp \in \Gamma$. Otherwise, $\Gamma$ is called open. The branch consisting of the initial formula (or formulas) to be tested for satisfiability is called the initial branch.

Let $\Gamma$ be a branch. With $\sim_{\Gamma}$ we denote the least equivalence relation $\sim$ on nominals such that $x \sim y$ for every equation $x \dot{=} y \in \Gamma$. Let $R(x, y)$ denote a term of the form $x \dot{=} y$, $x \neq y$, or $r x y$. We define the equational closure $\tilde{\Gamma}$ of a branch $\Gamma$ as

$$
\begin{aligned}
\tilde{\Gamma}:=\Gamma & \cup\left\{t x \mid t \text { modal expression } \wedge \exists x^{\prime}: x^{\prime} \sim_{\Gamma} x \wedge t x^{\prime} \in \Gamma\right\} \\
& \cup\left\{R(x, y) \mid \exists x^{\prime}, y^{\prime}: x^{\prime} \sim_{\Gamma} x \wedge y^{\prime} \sim_{\Gamma} y \wedge R\left(x^{\prime}, y^{\prime}\right) \in \Gamma\right\}
\end{aligned}
$$

Note that for all nominals $x$ and $y, x \sim_{\Gamma} y$ holds if and only if $x \doteq y \in \tilde{\Gamma}$. Since $\tilde{\Gamma}$ only contains nominals, modal expressions and roles that already occur on $\Gamma, \tilde{\Gamma}$ clearly is finite if $\Gamma$ is finite. Reasoning with respect to $\tilde{\Gamma}$ can be implemented efficiently using disjoint-set forests, as demonstrated in [14, 15]. 
3.2. Evidence. The proof of model existence for our calculus proceeds in two stages. Applied to a satisfiable initial branch, the rules of the calculus (defined in Sect. 3.3) construct a quasi-evident branch (defined in Sect. 3.4). We show that every quasi-evident branch can be extended to an evident branch. For evident branches, we show model existence. Intuitively, we call a branch evident if it contains a complete syntactic description of a model of all of its formulas.

We write $D_{\Gamma} X$ as an abbreviation for $\forall x, y \in X: x \neq y \Longrightarrow x \neq y \in \tilde{\Gamma} \vee y \neq x \in \tilde{\Gamma}$. A branch $\Gamma$ is called evident if it satisfies all of the following evidence conditions:

$$
\begin{aligned}
& \left(t_{1} \dot{\wedge} t_{2}\right) x \in \Gamma \Rightarrow t_{1} x \in \tilde{\Gamma} \wedge t_{2} x \in \tilde{\Gamma} \\
& \left(t_{1} \dot{\vee} t_{2}\right) x \in \Gamma \Rightarrow t_{1} x \in \tilde{\Gamma} \vee t_{2} x \in \tilde{\Gamma} \\
& \langle r\rangle_{n} t x \in \Gamma \Rightarrow \exists^{n+1} Y: D_{\Gamma} Y \wedge\{r x y, t y \mid y \in Y\} \subseteq \tilde{\Gamma} \\
& {[r]_{n} t x \in \Gamma \Rightarrow\left|\{y \mid r x y \in \tilde{\Gamma}, t y \notin \tilde{\Gamma}\} / \sim_{\Gamma}\right| \leq n} \\
& E_{n} t x \in \Gamma \Rightarrow \exists^{n+1} Y: D_{\Gamma} Y \wedge\{t y \mid y \in Y\} \subseteq \tilde{\Gamma} \\
& A_{n} t x \in \Gamma \Rightarrow\left|\{y \mid t y \notin \tilde{\Gamma}\} / \sim_{\Gamma}\right| \leq n \\
& \dot{x} y \in \Gamma \Rightarrow x \sim_{\Gamma} y \\
& \dot{\neg} \dot{x} y \in \Gamma \Rightarrow x \chi_{\Gamma} y \\
& x \neq y \in \Gamma \Rightarrow x \chi_{\Gamma} y \\
& \neg p x \in \Gamma \Rightarrow p x \notin \tilde{\Gamma}
\end{aligned}
$$

A formula $s$ is called evident on $\Gamma$ if $\Gamma$ satisfies the right-hand side of the evidence condition corresponding to $s$. For instance, $\left(t_{1} \dot{\wedge} t_{2}\right) x$ is evident on $\Gamma$ if and only if $\left\{t_{1} x, t_{2} x\right\} \subseteq \tilde{\Gamma}$.

Given a term $t$, we write $\mathcal{N} t$ for the set of nominals that occur in $t$. The notation is extended to sets of terms in the natural way: $\mathcal{N} \Gamma:=\bigcup\{\mathcal{N} t \mid t \in \Gamma\}$.

Theorem 3.1 (Model Existence). Every evident branch has a finite model.

Proof. Let $\Gamma$ be an evident branch and let $x_{0} \in \mathcal{N} \Gamma$. Let $\rho$ be a function from finite sets of nominals to nominals such that $\rho X \in X$ whenever $X$ is nonempty. We define the interpretation $\mathcal{I}$ such that:

$$
\begin{aligned}
& \mathcal{I S}:=\left\{\rho\left\{y \mid y \sim_{\Gamma} x\right\} \mid x \in \mathcal{N} \Gamma\right\} \\
& \mathcal{I} x:=\text { if } x \in \mathcal{N} \Gamma \text { then } \rho\left\{y \in \mathcal{N} \Gamma \mid y \sim_{\Gamma} x\right\} \text { else } \mathcal{I} x_{0} \\
& \mathcal{I} p:=\{x \in \mathcal{I} \mathrm{S} \mid p x \in \tilde{\Gamma}\} \\
& \mathcal{I} r:=\left\{(x, y) \in(\mathcal{I S})^{2} \mid r x y \in \tilde{\Gamma}\right\}
\end{aligned}
$$

Intuitively, we construct $\mathcal{I}$ by interpreting $\mathrm{S}$ as the quotient of the nominals on $\Gamma$ by $\sim_{\Gamma}$, where each equivalence class is represented by a fixed element of the class selected by $\rho$. Nominals on $\Gamma$ are mapped to their corresponding equivalence classes. All other nominals are mapped to some arbitrary state. Propositional variables and roles are interpreted as the smallest sets that are consistent with the respective assertions on $\Gamma$. Since $\Gamma$ is finite by definition, so is $\mathcal{I}$. Note that in the last two lines of the definition, we interpret the set notation as a convenient description for the respective characteristic functions.

We now show that, for all $s \in \Gamma, \mathcal{I}$ satisfies $s$ by induction on $s$. Let $s \in \Gamma$. We proceed by case analysis.

- $s=p x$. Since $\mathcal{I} x \sim_{\Gamma} x$, we have $p(\mathcal{I} x) \in \tilde{\Gamma}$. The claim follows. 
- $s=\dot{\neg} p x$. It suffices to show that $\mathcal{I}(p x)=0$. By the evidence condition for $s, p x \notin \tilde{\Gamma}$. Hence $p(\mathcal{I} x) \notin \tilde{\Gamma}$. The claim follows.

- $s=r x y$. Then $r(\mathcal{I} x)(\mathcal{I} y) \in \tilde{\Gamma}$, and hence $(\mathcal{I} x, \mathcal{I} y) \in \mathcal{I} r$.

- $s=x \dot{=}$. It suffices to show that $\mathcal{I} x=\mathcal{I} y$, which is the case as $x \sim_{\Gamma} y$ by the definition of $\sim \Gamma$.

- $s=x \neq y$. By the evidence condition for $s, x \chi_{\Gamma} y$. Hence $\mathcal{I}_{x} \chi_{\Gamma} \mathcal{I} y$. The claim follows.

- $s=\langle r\rangle_{n} t x$. By the evidence condition for $s$, there is a set $Y$ of cardinality $n+1$ such that $D_{\Gamma} Y$ and for all $y \in Y,\{r x y, t y\} \subseteq \tilde{\Gamma}$. By the inductive hypothesis for the disequations required by $D_{\Gamma} Y$, we have $\left|Y / \sim_{\Gamma}\right|=|\{\mathcal{I} y \mid y \in Y\}|=n+1$. By the inductive hypothesis for the formulas $r x y$ and $t y$ (for all $y \in Y$ ), we have $(\mathcal{I} x, \mathcal{I} y) \in \mathcal{I} r$, and $\mathcal{I}$ satisfies $t y$. The claim follows.

- $s=[r]_{n} t x$. By the evidence condition for $s,\left|\{y \mid r x y \in \tilde{\Gamma}, t y \notin \tilde{\Gamma}\} / \sim_{\Gamma}\right| \leq n$. Since $\mathcal{I} x \sim_{\Gamma} x$ whenever $x \in \mathcal{N} \Gamma$, we have for all $x, y \in \mathcal{N} \Gamma:(\mathcal{I} x, \mathcal{I} y) \in \mathcal{I} r \Leftrightarrow r(\mathcal{I} x)(\mathcal{I} y) \in$ $\tilde{\Gamma} \Leftrightarrow r x y \in \tilde{\Gamma}$. Hence $\left|\{y \mid r x y \in \tilde{\Gamma}, t y \notin \tilde{\Gamma}\} / \sim_{\Gamma}\right|=|\{\mathcal{I} y \mid(\mathcal{I} x, \mathcal{I} y) \in \mathcal{I} r, t y \notin \tilde{\Gamma}\}| \leq n$. Moreover, by the inductive hypothesis, $\mathcal{I}$ satisfies $t y$ whenever $t y \in \tilde{\Gamma}$. The claim follows. The cases $s=\left(t_{1} \dot{\vee} t_{2}\right) x, s=\left(t_{1} \dot{\wedge} t_{2}\right) x$ are straightforward. The cases $s=\dot{x} y$ and $s=\dot{\neg} \dot{x} y$ proceed analogously to $s=x \dot{=} y$ and, respectively, $s=x \neq y$, and the cases $s=E_{n} t x$ and $s=A_{n} t x$ are analogous but simpler than $s=\langle r\rangle_{n} t x$ and, respectively, $s=[r]_{n} t x$.

3.3. Tableau Rules. The tableau rules of our basic calculus $\mathcal{T}$ are defined in Fig. 1, In the rules, we write $\exists x \in X: \Gamma(x)$ for $\Gamma\left(x_{1}\right)|\ldots| \Gamma\left(x_{n}\right)$, where $X=\left\{x_{1}, \ldots, x_{n}\right\}$ and $\Gamma(x)$ is a set of formulas parameterized by $x$. In case $X=\emptyset$, the notation translates to $\perp$. Dually, we write $\forall x \in X: \Gamma(x)$ for $\Gamma\left(x_{1}\right), \ldots, \Gamma\left(x_{n}\right)\left(X=\left\{x_{1}, \ldots, x_{n}\right\}\right)$. If $X=\emptyset$, the notation stands for the empty set of formulas.

The side condition of $\mathcal{R} \diamond$ uses the notion of quasi-evidence that we will introduce in Sect. 3.4. For now, we assume the rule is formulated with the restriction " $\langle r\rangle_{n} t x$ not evident on $\Gamma$ ".

Note that for $n=0$, the rules $\mathcal{R}_{\diamond}$ and $\mathcal{R}_{\square}$ instantiate, modulo obvious simplifications, to their respective non-graded counterparts:

$$
\frac{\langle r\rangle_{0} t x}{r x y, t y} y \text { fresh, }\langle r\rangle_{0} t x \text { not quasi-evident on } \Gamma \quad \frac{[r]_{0} t x}{t y} r x y \in \tilde{\Gamma}
$$

A branch $\Delta$ is called a proper extension of a branch $\Gamma$ if $\Delta \supseteq \Gamma$ and $\tilde{\Delta} \supsetneq \tilde{\Gamma}$. Note that if $\Delta$ is a proper extension of $\Gamma$, in particular it holds $\Delta \supsetneq \Gamma$. The converse does not hold: Let $\Gamma:=\{\dot{x} y, x \doteq z, z \dot{=} y\}$ and $\Delta:=\Gamma \cup\{x \dot{=} y\}$. Then $\Delta \supsetneq \Gamma$ but $\Delta$ is not a proper extension of $\Gamma$. We implicitly restrict the applicability of the tableau rules so that a rule $\mathcal{R}$ is only applicable to a formula $s \in \Gamma$ if all of the alternative branches $\Delta_{1}, \ldots, \Delta_{n}$ resulting from this application are proper extensions of $\Gamma$. Moreover, we require that for every $i, j$ with $1 \leq i<j \leq n, \tilde{\Delta}_{i} \neq \tilde{\Delta}_{j}$. Whenever a rule produces several alternative branches whose equational closure is equal, by the following proposition it suffices to consider only one of them to preserve soundness.

Proposition 3.2. Let $\mathcal{I}$ be a modal interpretation and $\Gamma, \Delta$ be branches such that $\tilde{\Gamma}=\tilde{\Delta}$. Then $\mathcal{I}$ satisfies $\Gamma$ if and only if $\mathcal{I}$ satisfies $\Delta$. 


$$
\begin{aligned}
& \mathcal{R}_{\dot{\wedge}} \frac{(s \dot{\wedge} t) x}{s x, t x} \quad \mathcal{R}_{\dot{\vee}} \frac{(s \dot{\vee} t) x}{s x \mid t x} \\
& \mathcal{R} \diamond \frac{\langle r\rangle_{n} t x}{\forall y \in Y: r x y, t y, \forall z \in Y, y \neq z: y \neq z} Y \text { fresh, }|Y|=n+1,\langle r\rangle_{n} t x \text { not quasi-evident on } \Gamma \\
& \mathcal{R}_{\square} \frac{[r]_{n} t x}{\exists y, z \in Y, y \neq z: y \dot{=} z \mid \exists y \in Y: t y} Y \subseteq\{y \mid r x y \in \tilde{\Gamma}\},|Y|=\left|Y / \sim_{\Gamma}\right|=n+1 \\
& \mathcal{R}_{E} \frac{E_{n} t x}{\forall y \in Y: t y, \forall z \in Y, y \neq z: y \neq z} Y \text { fresh, }|Y|=n+1, E_{n} t x \text { not evident on } \Gamma \\
& \mathcal{R}_{A} \frac{A_{n} t x}{\exists y, z \in Y, y \neq z: y \doteq z \mid \exists y \in Y: t y} Y \subseteq \mathcal{N} \Gamma,|Y|=\left|Y / \sim_{\Gamma}\right|=n+1 \\
& \mathcal{R}_{N} \frac{\dot{x} y}{x \dot{=} y} \quad \mathcal{R}_{\bar{N}} \frac{\dot{\neg} \dot{x} y}{x \neq y} \quad \mathcal{R}_{\dot{\lrcorner}}^{\perp} \frac{\dot{\lrcorner} p x}{\perp} p x \in \tilde{\Gamma} \quad \mathcal{R}_{\neq}^{\perp} \frac{x \neq y}{\perp} x \sim_{\Gamma} y
\end{aligned}
$$

$\Gamma$ is the branch to which a rule is applied.

" $Y$ fresh" stands for $Y \cap \mathcal{N} \Gamma=\emptyset$.

Figure 1: Tableau rules for $\mathcal{T}$

Proposition 3.3 (Soundness). Let $\Delta_{1}, \ldots, \Delta_{n}$ be the branches obtained from a branch $\Gamma$ by a rule of $\mathcal{T}$. Then $\Gamma$ is satisfiable if and only if there is some $i \in\{1, \ldots, n\}$ such that $\Delta_{i}$ is satisfiable.

Example 3.4. Consider the unsatisfiable formula $\left(\langle r\rangle_{1} p \dot{\wedge}[r]_{1} \dot{\neg} p\right) x$. Applied to the formula, our tableau rules produce three closed branches as shown in Fig. 2, All the rule applications except $\mathcal{R}_{\square}$ produce exactly one extension. The rule $\mathcal{R}_{\square}$ applies to the formula $[r]_{1} \dot{\neg} p x$ and the set $Y=\{y, z\}$ producing three extensions. The leftmost branch is closed with $\mathcal{R}_{\neq}^{\perp}$ applied to $y \neq z$, the other two branches are closed with $\mathcal{R}_{\dot{\rightarrow}}^{\perp}$ applied to the respective two formulas introduced by the application of $\mathcal{R}_{\square}$. Note that without the restriction that the equational closures of alternative extensions must be different the application of $\mathcal{R}_{\square}$ would introduce an additional fourth extension, namely by the equation $z \doteq y$.

3.4. Control. The restrictions on the applicability of the tableau rules given by the evidence conditions are not sufficient for termination. Consider $\Gamma_{0}:=\left\{A_{0}\langle r\rangle_{0} p x\right\}$. An application of $\mathcal{R}_{A}$ to $\Gamma_{0}$ yields $\Gamma_{1}:=\Gamma_{0} \cup\left\{\langle r\rangle_{0} p x\right\}$, which can be extended by $\mathcal{R}_{\diamond}$ to $\Gamma_{2}:=\Gamma_{1} \cup\{r x y, p y\}$. Now $\mathcal{R}_{A}$ is applicable again and yields $\Gamma_{3}:=\Gamma_{2} \cup\left\{\langle r\rangle_{0} p y\right\}$, which in turn can be extended by $\mathcal{R}_{\diamond}$, and so ad infinitum.

To obtain a terminating calculus, the rule $\mathcal{R}_{\diamond}$ needs to be restricted further. We do so by weakening the notion of evidence for diamond formulas. The weaker notion, called 


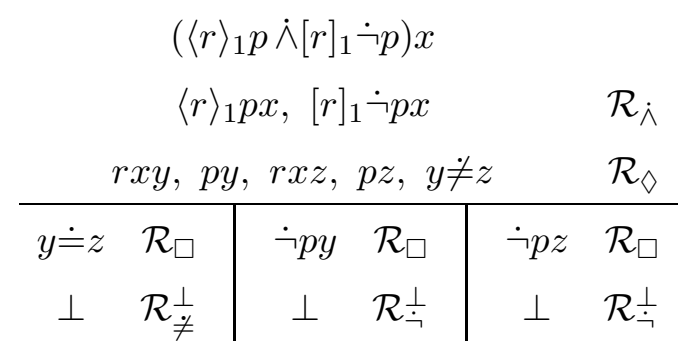

Figure 2: Tableau derivation for $\left(\langle r\rangle_{1} p \dot{\wedge}[r]_{1} \dot{\neg} p\right) x$

quasi-evidence, is then used in the side condition of $\mathcal{R}_{\diamond}$ in place of evidence. As we have mentioned before, an evident branch contains a complete description of a model of all of its formulas. A quasi-evident branch will contain only a partial description of such a model. In particular, quasi-evidence will not require that for every diamond $\langle r\rangle_{n} t x$, we have $n+1$ outgoing edges rxy. However, we require that the partial description given by a quasievident branch can always be completed to a full model of the branch by adding edges. So, in particular, every quasi-evident branch will be satisfiable. In the above example, $\Gamma_{3}$ will turn out to be quasi-evident and hence terminal. And indeed, $\Gamma_{3}$ is clearly satisfiable and can be completed to an evident branch by adding the edge ryy.

While quasi-evidence was introduced in the context of pattern-based blocking, it can also be made sense of in the context of chain-based blocking. Unlike with pattern-based blocking, calculi using chain-based blocking usually terminate with branches that are not quasi-evident, which is due to the presence of "blocked" parts, i.e., parts of the branch that have at some point been identified as irrelevant for the model construction and so have been excluded from further processing. The parts that are not blocked form a kernel from which a model can be constructed. And in many cases, this kernel is precisely what we call a quasi-evident branch. A concrete example relating chain-based blocking and quasi-evidence is given in $[24$.

Our task is now to define a notion of quasi-evidence that is weak enough to guarantee termination of our calculus but strong enough to preserve completeness in the presence of graded modalities. The notions of quasi-evidence used in previous work on pattern-based blocking [23, 24 turn out to be too weak. For instance, intuitively adapting the notion in 23 would give us the following candidate definition:

A formula $\langle r\rangle_{m} s x$ is quasi-evident on $\Gamma$ if there are nominals $y, z_{1}, \ldots, z_{m+1}$ such that $\left\{r y z_{1}, s z_{1}, \ldots, r y z_{m+1}, s z_{m+1}\right\} \subseteq \tilde{\Gamma}$ and $\left\{[r]_{n} t y \mid[r]_{n} t x \in \tilde{\Gamma}\right\} \subseteq \tilde{\Gamma}$. (We also say: $\langle r\rangle_{m} s x$ is quasi-evident if the corresponding pattern $\left\{\langle r\rangle_{m} s\right\} \cup\left\{[r]_{n} t \mid[r]_{n} t x \in \tilde{\Gamma}\right\}$ is expanded).

With this definition of quasi-evidence, no rule of our calculus would apply to the following branch:

$$
\Gamma:=\left\{r y z, q z,[r]_{1}(p \dot{\wedge} \dot{\neg} p) y,\langle r\rangle_{0} q x,[r]_{1}(p \dot{\wedge} \dot{\neg} p) x, r x u, \dot{\neg} q u\right\}
$$

As $\Gamma$ is clearly unsatisfiable, the notion of quasi-evidence needs to be adapted.

Given a branch $\Gamma$ and a role $r$, an $r$-pattern is a set of expressions of the form $\mu s$, where $\mu \in\left\{\langle r\rangle_{n},[r]_{n} \mid n \in \mathbb{N}\right\}$. We write $P_{\Gamma}^{r} x$ for the largest $r$-pattern $P$ such that $P \subseteq\{t \mid t x \in \tilde{\Gamma}\}$. We call $P_{\Gamma}^{r} x$ the $r$-pattern of $x$ on $\Gamma$. An $r$-pattern $P$ is expanded on $\Gamma$ if there are nominals $x, y$ such that $r x y \in \tilde{\Gamma}$ and $P \subseteq P_{\Gamma}^{r} x$. In this case, we say that the nominal $x$ expands $P$ on $\Gamma$. 
A diamond formula $\langle r\rangle_{n} s x \in \Gamma$ is quasi-evident on $\Gamma$ if it is either evident on $\Gamma$ or $x$ has no $r$-successor on $\Gamma$ (i.e., there is no $y$ such that $r x y \in \tilde{\Gamma}$ ) and $P_{\Gamma}^{r} x$ is expanded on $\Gamma$. The rule $\mathcal{R}_{\diamond}$ can only be applied to diamond formulas that are not quasi-evident.

Note that whenever $\langle r\rangle_{n} s x \in \Gamma$ is quasi-evident but not evident on $\Gamma$, there is a nominal $y$ that expands $P_{\Gamma}^{r} x$ on $\Gamma$.

We call a branch $\Gamma$ quasi-evident if it satisfies all of the evidence conditions but the one for diamond formulas, which we replace by:

$$
\langle r\rangle_{n} t x \in \Gamma \Rightarrow\langle r\rangle_{n} t x \text { is quasi-evident on } \Gamma
$$

Example 3.5. Figure 3 shows a tableau derivation resulting in a quasi-evident branch. Let us write $\Gamma_{n}$ for the branch obtained in line $n$ of the derivation. Note that $P_{\Gamma_{3}}^{r} x=$ $\left\{\langle r\rangle_{0} p,\langle r\rangle_{0} q\right\}$ is expanded on $\Gamma_{3}$. The notion of expandedness is such that, once expanded, a pattern remains expanded on all extensions of the branch. In particular, if $P_{\Gamma_{i}}^{r} x$ is expanded on $\Gamma_{i}$, then $P_{\Gamma_{i}}^{r} x$ (not, however, $P_{\Gamma_{j}}^{r} x$ ) will be expanded on $\Gamma_{j}$ for all $j \geq i$. Note that the pattern of a nominal may change over time, i.e., $P_{\Gamma_{i}}^{r} x$ and $P_{\Gamma_{j}}^{r} x$ may be different if $i \neq j$. So, in the example, $P_{\Gamma_{1}}^{r} x=\emptyset \subsetneq P_{\Gamma_{3}}^{r} x$. In general, we have $P_{\Gamma_{i}}^{r} x \subseteq P_{\Gamma_{j}}^{r} x$ whenever $i \leq j$. However, if $x \sim_{\Gamma_{i}} y$ and $x$ expands $P_{\Gamma_{i}}^{r} y$ on $\Gamma_{i}$, then $x$ will expand $P_{\Gamma_{j}}^{r} y$ on $\Gamma_{j}$ for all $j \geq i$.

Since $P_{\Gamma_{5}}^{r} x=P_{\Gamma_{5}}^{r} y, P_{\Gamma_{5}}^{r} y$ is expanded on $\Gamma_{5}$, and hence both $\langle r\rangle_{0} p y$ and $\langle r\rangle_{0} q y$ are quasievident on $\Gamma_{5}$. The pattern $P_{\Gamma_{5}}^{r^{\prime}} y=\left\{\left\langle r^{\prime}\right\rangle_{0} q\right\}$ is not expanded on $\Gamma_{5}$, so $\mathcal{R}_{\diamond}$ is applicable to $\left\langle r^{\prime}\right\rangle_{0} q y$. On the branch $\Gamma_{6}$ resulting from this application, the pattern becomes expanded, and so does $P_{\Gamma_{6}}^{r^{\prime}} x$. The only diamond formula that is not quasi-evident on $\Gamma_{6}$ is $\langle r\rangle_{0} q x$ (since it is not evident and $x$ has a successor on $\Gamma_{6}$ ). After applying $\mathcal{R}_{\diamond}$ to $\langle r\rangle_{0} q x, \Gamma_{7}$ contains only quasi-evident diamond formulas. To make the branch evident, it remains to propagate the universal constraint $\langle r\rangle_{0} p \dot{\wedge}\langle r\rangle_{0} q \dot{\wedge}\left\langle r^{\prime}\right\rangle_{0} q$ to $z$ and $u$ (steps 8-11). Since this introduces no new patterns (we have $P_{\Gamma_{11}}^{r} z=P_{\Gamma_{11}}^{r} u=P_{\Gamma_{11}}^{r} y=P_{\Gamma_{11}}^{r} x$ and $P_{\Gamma_{11}}^{r^{\prime}} z=P_{\Gamma_{11}}^{r^{\prime}} u=P_{\Gamma_{11}}^{r^{\prime}} x=$ $\left.P_{\Gamma_{11}}^{r^{\prime}} y\right), \Gamma_{11}$ is quasi-evident.

Lemma 3.6. Let $\Gamma$ be a quasi-evident branch and let $\langle r\rangle_{n} s x \in \Gamma$ be not evident on $\Gamma$. Let $y$ be a nominal that expands $P_{\Gamma}^{r} x$ on $\Gamma$ and let $\Delta:=\Gamma \cup\{r x z \mid r y z \in \tilde{\Gamma}\}$. Then:

(1) $\forall z: r x z \in \tilde{\Delta} \Longleftrightarrow r y z \in \tilde{\Gamma}$,

(2) $\forall m, t:\langle r\rangle_{m} t \in P_{\Gamma}^{r} x \Longrightarrow\langle r\rangle_{m} t x$ evident on $\Delta$,

(3) $\langle r\rangle_{n}$ sx evident on $\Delta$,

(4) $\forall r^{\prime}, m, t, z:\left\langle r^{\prime}\right\rangle_{m} t z$ evident on $\Gamma \Longrightarrow\left\langle r^{\prime}\right\rangle_{m} t z$ evident on $\Delta$,

(5) $\Delta$ quasi-evident.

Proof. We begin with (1). Let $z$ be a nominal. By construction, it holds $r y z \in \tilde{\Gamma} \Rightarrow r x z \in$ $\Delta$. The converse implication holds by the fact that $\langle r\rangle_{n} s x$ is quasi-evident but not evident on $\Gamma$, meaning that $x$ has no $r$-successor on $\Gamma$. It remains to show: $r x z \in \Delta \Leftrightarrow r x z \in \tilde{\Delta}$. The direction from left to right is obvious. For the other direction, assume $r x z \in \tilde{\Delta}$. Then there are $x^{\prime}, z^{\prime}$ such that $x^{\prime} \sim_{\Gamma} x, z^{\prime} \sim_{\Gamma} z$, and $r x^{\prime} z^{\prime} \in \Delta$. Since $x$ has no $r$-successor on $\Gamma$, neither does $x^{\prime}$. Hence, by the definition of $\Delta$, we must have $x^{\prime}=x$, and so $r x z^{\prime} \in \Delta$. But then $r y z^{\prime} \in \tilde{\Gamma}$, and consequently $r y z \in \tilde{\Gamma}$. The claim follows by the definition of $\Delta$.

Now to (2). Let $\langle r\rangle_{m} t \in P_{\Gamma}^{r} x$. Since $P_{\Gamma}^{r} y \supseteq P_{\Gamma}^{r} x$, in particular it holds $\langle r\rangle_{m} t y \in \tilde{\Gamma}$, i.e., there is some $y^{\prime} \sim_{\Gamma} y$ such that $\langle r\rangle_{m} t y^{\prime} \in \Gamma$. By (1), it suffices to show that $\langle r\rangle_{m} t y$ is evident on $\Gamma$. This is the case since $\langle r\rangle_{m} t y^{\prime}$ is quasi-evident on $\Gamma$ (as $\Gamma$ is quasi-evident) and $y^{\prime}$ has an $r$-successor on $\Gamma$ (as $y$ has one on $\Gamma$ ). 


$\begin{array}{lcl}\text { 0. } & A_{0}\left(\langle r\rangle_{0} p \dot{\wedge}\langle r\rangle_{0} q \dot{\wedge}\left\langle r^{\prime}\right\rangle_{0} q\right) x & \\ \text { 1. } & \left(\langle r\rangle_{0} p \dot{\wedge}\langle r\rangle_{0} q \dot{\wedge}\left\langle r^{\prime}\right\rangle_{0} q\right) x & \mathcal{R}_{A} \\ \text { 2. } & \left(\langle r\rangle_{0} p \dot{\wedge}\langle r\rangle_{0} q\right) x,\langle r\rangle_{0} p x,\langle r\rangle_{0} q x,\left\langle r^{\prime}\right\rangle_{0} q x & 2 \times \mathcal{R}_{\dot{\wedge}} \\ \text { 3. } & r x y, p y & \mathcal{R}_{\diamond} \\ \text { 4. } & \left(\langle r\rangle_{0} p \dot{\wedge}\langle r\rangle_{0} q \dot{\wedge}\left\langle r^{\prime}\right\rangle_{0} q\right) y & \mathcal{R}_{A} \\ \text { 5. } & \left(\langle r\rangle_{0} p \dot{\wedge}\langle r\rangle_{0} q\right) y,\langle r\rangle_{0} p y,\langle r\rangle_{0} q y,\left\langle r^{\prime}\right\rangle_{0} q y & 2 \times \mathcal{R}_{\dot{\wedge}} \\ \text { 6. } & r^{\prime} y z, q z & \mathcal{R}_{\diamond} \\ \text { 7. } & r x u, q u & \mathcal{R}_{\diamond} \\ \text { 8. } & \left(\langle r\rangle_{0} p \dot{\wedge}\langle r\rangle_{0} q \dot{\wedge}\left\langle r^{\prime}\right\rangle_{0} q\right) z & \mathcal{R}_{A} \\ \text { 9. } & \left(\langle r\rangle_{0} p \dot{\wedge}\langle r\rangle_{0} q\right) z,\langle r\rangle_{0} p z,\langle r\rangle_{0} q z,\left\langle r^{\prime}\right\rangle_{0} q z & 2 \times \mathcal{R}_{\dot{\Lambda}} \\ \text { 10. } & \left(\langle r\rangle_{0} p \dot{\wedge}\langle r\rangle_{0} q \dot{\wedge}\left\langle r^{\prime}\right\rangle_{0} q\right) u & \mathcal{R}_{A} \\ \text { 11. } & \left(\langle r\rangle_{0} p \dot{\wedge}\langle r\rangle_{0} q\right) u,\langle r\rangle_{0} p u,\langle r\rangle_{0} q u,\left\langle r^{\prime}\right\rangle_{0} q u & 2 \times \mathcal{R}_{\dot{\Lambda}}\end{array}$

Figure 3: Tableau derivation for $A_{0}\left(\langle r\rangle_{0} p \dot{\wedge}\langle r\rangle_{0} q \dot{\wedge}\left\langle r^{\prime}\right\rangle_{0} q\right) x$

Claim (3) immediately follows from (2).

Claim (4) is obvious as the evidence of diamonds on a branch cannot be destroyed by adding edges.

Now to (5). The only conditions that might in principle be violated on $\Delta$ are the quasievidence condition for diamonds of the form $\langle r\rangle_{m} t z \in \Delta$ where $z \sim_{\Delta} x$, and the evidence condition for boxes $[r]_{m} t z \in \Delta$ where $z \sim_{\Delta} x$.

For diamonds of the above form, the quasi-evidence condition holds by (2).

If $[r]_{m} t z \in \Delta$ and $z \sim_{\Delta} x$, it holds $[r]_{m} t y \in \tilde{\Gamma}$ since $P_{\Gamma}^{r} y \supseteq P_{\Gamma}^{r} x=P_{\Delta}^{r} x$. Hence by $(1)$ it suffices to show that $[r]_{m}$ ty is evident on $\Gamma$, which is the case since $\Gamma$ is quasi-evident.

Theorem 3.7 (Evidence Completion). For every quasi-evident branch $\Gamma$ there is an evident branch $\Delta$ such that $\Gamma \subseteq \Delta$.

Proof. For every branch $\Gamma$ we define:

$$
\varphi \Gamma:=\mid\left\{\langle r\rangle_{n} s x \mid\langle r\rangle_{n} s x \in \Gamma \wedge\langle t\rangle_{n} s x \text { not evident on } \Gamma\right\} \mid
$$

Let $\Gamma$ be quasi-evident. We proceed by induction on $\varphi \Gamma$. If $\varphi \Gamma=0$, then $\Gamma$ is evident and we are done. Otherwise, there is a diamond $\langle r\rangle_{n} s x \in \Gamma$ that is not evident on $\Gamma$. Let $y$ be a nominal that expands $P_{\Gamma}^{r} x$ on $\Gamma$, and let $\Gamma^{\prime}:=\Gamma \cup\{r x z \mid r y z \in \tilde{\Gamma}\}$. By Lemma 3.6(3-5), $\Gamma^{\prime}$ is quasi-evident and $\varphi \Gamma^{\prime}<\varphi \Gamma$. So, by the inductive hypothesis, there is some evident branch $\Delta$ such that $\Gamma \subseteq \Gamma^{\prime} \subseteq \Delta$.

A branch is called maximal if it cannot be extended by any tableau rule.

Theorem 3.8 (Quasi-evidence). Every open and maximal branch in $\mathcal{T}$ is quasi-evident.

Proof. Let $\Gamma$ be an open and maximal branch. Note that we have no evidence or quasievidence conditions for formulas of the form $p x, r x y$ or $x \doteq y$. We show that every $s \in \Gamma$ 
that is not of the form $p x, r x y$ or $x \doteq y$ is (quasi-)evident on $\Gamma$ by case analysis on the shape of $s$.

- $s=\dot{\neg} x$. The claim, $p x \notin \tilde{\Gamma}$, follows by $\mathcal{R}_{\dot{\neg}}^{\perp}$ (and the assumption that $\Gamma$ is open and maximal).

- $s=x \neq y$. The claim, $x \varkappa_{\Gamma} y$, follows by $\mathcal{R}_{\neq}^{\perp}$ (and the assumption that $\Gamma$ is open and maximal).

- $s=\dot{x} y$. By $\mathcal{R}_{N}, x \doteq y \in \tilde{\Gamma}$ and hence $x \sim_{\Gamma} y$.

- $s=\dot{\neg} \dot{x} y$. By $\mathcal{R}_{\bar{N}}, x \neq y \in \tilde{\Gamma}$. Then there are some $x^{\prime}$ and $y^{\prime}$ such that $x^{\prime} \sim_{\Gamma} x, y^{\prime} \sim_{\Gamma} y$, and $x^{\prime} \neq y^{\prime} \in \Gamma$. By $\mathcal{R} \underset{\neq}{\perp}$, we have $x^{\prime} \chi_{\Gamma} y^{\prime}$ (cf. $s=x \neq y$ ). The claim follows by the transitivity of $\sim_{\Gamma}$.

- $s=[r]_{n} t x$. To show: $\left|\{y \mid r x y \in \tilde{\Gamma}, t y \notin \tilde{\Gamma}\} / \sim_{\Gamma}\right| \leq n$. This is clearly the case if $|\{y \mid r x y \in \tilde{\Gamma}\}| \leq n$. Otherwise, it suffices to show that for every $Y \subseteq\{y \mid r x y \in \tilde{\Gamma}\}$ such that $|Y|=n+1$, it either holds $\left|Y / \sim_{\Gamma}\right|<|Y|$ or $t y \in \tilde{\Gamma}$ for some $y \in Y$. This follows by $\mathcal{R}_{\square}$ since $y \doteq z \in \Gamma$ implies $y \sim_{\Gamma} z$ for all $y, z \in Y$.

The cases $s=\left(t_{1} \dot{\vee} t_{2}\right) x, s=\left(t_{1} \dot{\wedge} t_{2}\right) x$, and $s=\langle r\rangle_{n} t x$ are immediate by, respectively, $\mathcal{R}_{\dot{\vee}}$, $\mathcal{R}_{\dot{\wedge}}$, and $\mathcal{R}_{\diamond}$. The cases $s=E_{n} t x$ and $s=A_{n} t x$ are proved analogously to $s=\langle r\rangle_{n} t x$ and, respectively, $s=[r]_{n} t x$.

3.5. Termination. We will now show that every tableau derivation is finite. As usual, the main difficulty is bounding the number of applications of generative rules, in particular of $\mathcal{R}_{\diamond}$. The present proof is notably more complex than the proofs in [23, 24] since now, an application of $\mathcal{R}_{\diamond}$ does not necessarily expand a new pattern. Hence, we need to combine the pattern-counting argument from [23, 24] with a bound on the number of non-expanding applications of $\mathcal{R}_{\diamond}$.

Since the rules $\mathcal{R}_{\dot{\vee}}, \mathcal{R}_{\square}$, and $\mathcal{R}_{A}$ are all finitely branching, by König's lemma it suffices to show that the construction of every individual branch terminates. Since tableau rule application always produces proper extensions of branches, it then suffices to show that the size (i.e., cardinality) of an individual branch is bounded.

First, we show that the size of a branch $\Gamma$ is bounded by a function in the number of nominals on $\Gamma$. Then, we show that this number itself is bounded from above, completing the termination proof.

We write $\Gamma \stackrel{\mathcal{R}}{\rightarrow} \Delta$ to denote that the branch $\Delta$ is obtained from $\Gamma$ by the rule $\mathcal{R}$. We write $\Gamma \rightarrow \Delta$ if $\Delta$ is obtained from $\Gamma$ by a single rule application. We write $\mathcal{S} \Gamma$ for the set of all modal expressions occurring on $\Gamma$, possibly as subterms of other expressions, and Rel $\Gamma$ for the set of all roles that occur on $\Gamma$.

Crucial for the termination argument is the fact the tableau rules cannot introduce any modal expressions that do not already occur on the initial branch.

Proposition 3.9. If $\Gamma, \Delta$ are branches such that $\Delta$ is obtained from $\Gamma$ by any rule of $\mathcal{T}$, then $\mathcal{S} \Delta=\mathcal{S} \Gamma$.

For every pair of nominals $x, y$ a branch $\Gamma$ may contain an equation $x \doteq y$ or a disequation $x \neq y$. For every pair $x, y$ and every role $r, \Gamma$ may contain an edge $r x y$. Moreover, for every expression $s \in \mathcal{S} \Gamma, \Gamma$ may contain a formula $s x$. Hence, the size of $\Gamma$ is bounded by $(2+|\operatorname{Rel} \Gamma|) \cdot|\mathcal{N} \Gamma|^{2}+|\mathcal{S} \Gamma| \cdot|\mathcal{N} \Gamma|$. By Proposition 3.9, we know that $|\mathcal{S} \Gamma|$ and $|\operatorname{Rel} \Gamma|$ depend 
only on the initial branch. Clearly, $|\mathcal{S} \Gamma|$ and $|\operatorname{Rel} \Gamma|$ are bounded from above by the size of the input, i.e., the sum of the sizes of the initial formulas.

By the above, it suffices to show that $|\mathcal{N} \Gamma|$ is exponentially bounded in the size of the input. We do so by giving a bound on the number of applications of $\mathcal{R}_{\diamond}$ and $\mathcal{R}_{E}$ that can occur in the derivation of a branch, which suffices since $\mathcal{R}_{\diamond}$ and $\mathcal{R}_{E}$ are the only two rules that can introduce new nominals.

We begin by showing that $\mathcal{R}_{E}$ can be applied at most as many times as there are distinct modal expressions of the form $E_{n} s$ on the initial branch. For this purpose, we define a function $\psi_{E}$ such that $\psi_{E} \Gamma:=\left\{E_{n} s \in \mathcal{S} \Gamma \mid \exists x \in \mathcal{N} \Gamma: E_{n} s x\right.$ not evident on $\left.\Gamma\right\}$. Since $\left|\psi_{E} \Gamma\right|$ is bounded from below by 0 , it suffices to show that the number decreases with every application of $\mathcal{R}_{E}$ (and is non-increasing otherwise, which is obvious).

Lemma 3.10. Let $s$ be of the form $\langle r\rangle_{n} t x$ or $E_{n} t x$. If $s$ is evident on $\Gamma$ and $\Gamma \subseteq \Delta$, then $s$ is evident on $\Delta$.

Proposition 3.11. $\Gamma \stackrel{\mathcal{R}_{E}}{\rightarrow} \Delta \Longrightarrow\left|\psi_{E} \Gamma\right|>\left|\psi_{E} \Delta\right|$

Proof. Let $\Gamma \stackrel{\mathcal{R}_{E}}{\rightarrow} \Delta$. By Lemma 3.10, $\psi_{E} \Gamma \supseteq \psi_{E} \Delta$. Hence it suffices to show that $\psi_{E} \Gamma-\psi_{E} \Delta$ is non-empty. Let $\Delta$ be obtained from $\Gamma$ by applying $\mathcal{R}_{E}$ to $s=E_{n} t x$. Then, by $\mathcal{R}_{E}$, $E_{n} t \in \psi_{E} \Gamma$. On the other hand, $s$ is evident on $\Delta$, and it is easy to see that the evidence of $s$ implies the evidence of $E_{n} t y$ for every $y \in \mathcal{N} \Delta$. Hence $E_{n} t \notin \psi_{E} \Delta$.

Now we show that $\mathcal{R}_{\diamond}$ can be applied at most finitely often in a derivation. Since there are only finitely many roles, it suffices to show that $\mathcal{R}_{\diamond}$ can be applied at most finitely often for each role. Observe that since $\mathcal{R}_{\diamond}$ is only applicable to diamond formulas that are not quasi-evident, it holds:

Proposition 3.12. If $\mathcal{R}_{\diamond}$ is applicable to a formula $\langle r\rangle_{n} s x \in \Gamma$, then either

(1) $x$ has an $r$-successor on $\Gamma$, or

(2) $P_{\Gamma}^{r} x$ is not expanded on $\Gamma$.

Let $\Gamma$ and $\Delta$ be branches such that $\Delta$ is obtained from $\Gamma$ by applying $\mathcal{R}_{\diamond}$ to a formula $\langle r\rangle_{n} s x \in \Gamma$ such that $P_{\Gamma}^{r} x$ is not expanded on $\Gamma$. It is easy to see that $P_{\Delta}^{r} x$ must be expanded on $\Delta$. Let us call such an application of $\mathcal{R}_{\diamond}$ pattern-expanding.

Let $\operatorname{Pat}^{r} \Gamma:=\mathcal{P}\left(\left\{\langle r\rangle_{n} s \in \mathcal{S} \Gamma\right\} \cup\left\{[r]_{n} s \in \mathcal{S} \Gamma\right\}\right)$. In other words, Pat $^{r} \Gamma$ contains all the possible sets of $r$-diamonds and $r$-boxes from $\mathcal{S} \Gamma$. Since $\Gamma \rightarrow \Delta$ implies $\tilde{\Gamma} \subseteq \tilde{\Delta}$, it holds:

Lemma 3.13. Let $\Gamma \rightarrow \Delta$ and $P \in$ Pat $^{r} \Gamma$. If $P$ is expanded on $\Gamma$, then $P$ is expanded on $\Delta$.

So, for each role $r$ the derivation of a branch has at most $\left|\operatorname{Pat}^{r} \Gamma_{0}\right|$ pattern-expanding applications of $\mathcal{R}_{\diamond}$, where $\Gamma_{0}$ is the initial branch. Clearly, $\left|\mathrm{Pat}^{r} \Gamma_{0}\right|$ is exponentially bounded in the size of the input.

Hence, it remains to show that a derivation can contain only finitely many applications of $\mathcal{R}_{\diamond}$ assuming that none of the applications is pattern-expanding. We say a nominal $x$ has a successor on $\Gamma$ if $x$ has an $r$-successor on $\Gamma$ for any role $r$. A set of nominals $X$ has a successor on $\Gamma$ if there is some $x \in X$ that has a successor on $\Gamma$. We define

$$
\psi_{\diamond}^{X} \Gamma:=\mid\left\{\langle r\rangle_{n} s \in \mathcal{S} \Gamma \mid \exists x \in X:\langle r\rangle_{n} s x \text { not evident on } \Gamma\right\} \mid
$$


and

$$
\psi_{\diamond} \Gamma:=\sum_{\substack{X \in \mathcal{N} \Gamma / \sim_{\Gamma} \\ X \text { has a successor on } \Gamma}} \psi_{\diamond}^{X} \Gamma .
$$

Lemma 3.14. Let $X, Y \in \mathcal{N} \Gamma / \sim_{\Gamma}, x \in X, y \in Y$, and let $\Delta:=\Gamma \cup\{x \doteq y\}$. Then $\psi_{\diamond}^{X} \Gamma \geq \psi_{\diamond}^{X \cup Y} \Delta$.

Proposition 3.15. Let $\Gamma \rightarrow \Delta$ such that $\Delta$ is obtained from $\Gamma$ by some rule application other than a pattern-expanding application of $\mathcal{R}_{\diamond}$.

(1) If $\Delta$ is obtained from $\Gamma$ by $\mathcal{R}_{\diamond}$, then $\psi_{\diamond} \Gamma>\psi_{\diamond} \Delta$.

(2) Otherwise, $\psi_{\diamond} \Gamma \geq \psi_{\diamond} \Delta$.

Proof.

(1) Clearly, nominals introduced by $\mathcal{R}_{\diamond}$ are fresh and hence cannot have any successors on $\Delta$. Hence $\psi_{\diamond} \Gamma \geq \psi_{\diamond} \Delta$. Therefore, it suffices to find a set $X \in \mathcal{N} \Gamma / \sim_{\Gamma}$ that has a successor on $\Gamma$, a nominal $x \in X$ and a formula $\langle r\rangle_{n} s x \in \Gamma$ that is not evident on $\Gamma$ but is evident on $\Delta$.

Assume $\Delta$ is obtained from $\Gamma$ by $\mathcal{R}_{\diamond}$ applied to a formula $\langle r\rangle_{n} s x \in \Gamma$. Clearly, $\langle r\rangle_{n} s x$ is not evident on $\Gamma$ but is evident on $\Delta$. Since the rule application is not pattern-expanding, $x$ has an $r$-successor on $\Gamma$. Hence there is some $X \in \mathcal{N} \Gamma / \sim_{\Gamma}$ such that $x \in X$ and $X$ has a successor on $\Gamma$. The claim follows.

(2) Since cumulativity of tableau construction preserves the evidence of diamond formulas (Lemma 3.10), the only interesting rules are those modifying $\mathcal{N} \Gamma / \sim_{\Gamma}$. Nominals introduced by $\mathcal{R}_{E}$ are fresh and hence do not have any successors on $\Delta$. Therefore, the only remaining cases are $\mathcal{R}_{N}, \mathcal{R}_{\square}$ and $\mathcal{R}_{A}$. Clearly, none of the three rules can increase the cardinality of $\left\{X \in \mathcal{N} \Gamma / \sim_{\Gamma} \mid X\right.$ has a successor on $\left.\Gamma\right\}$. The claim follows by Lemma 3.14 .

This completes the termination proof. Since the cardinalities of the sets $\operatorname{Pat}^{r} \Gamma$ are exponentially bounded in the size $n_{0}$ of the input, $\left|\psi_{E} \Gamma\right|$ is polynomial in $n_{0}$, and $\psi_{\diamond} \Gamma$ polynomial in $|\Gamma|$ and $n_{0},|\mathcal{N} \Gamma|$ is exponentially bounded in $n_{0}$. Since $|\Gamma|$ is polynomial in $|\mathcal{N} \Gamma|$, we conclude that $|\Gamma|$ is at most exponential in $n_{0}$. By cumulativity, the construction of $\Gamma$ terminates in at most exponentially many steps in $n_{0}$. This suffices to give us a NExPTIme complexity bound for the decision procedure based on the calculus.

\section{Adding Reflexivity, Transitivity and Role Inclusion}

We now extend $\mathcal{T}$ to deal with reflexivity, transitivity and inclusion assertions. As in related work on description logic [16, 20, 18, 19, 17, we restrict our modal expressions to contain no graded boxes for roles that have transitive subroles.

We define $\subseteq_{\Gamma}^{*}$ as the smallest reflexive and transitive relation such that $r \subseteq_{\Gamma}^{*} r^{\prime}$ whenever $r \sqsubseteq r^{\prime} \in \Gamma$. A role $r$ is called simple on a branch $\Gamma$ (or just simple if $\Gamma$ is clear from the context) if there is no $r^{\prime}$ such that $r^{\prime} \subseteq_{\Gamma}^{*} r$ and $T r^{\prime} \in \Gamma$. Observe that all subroles of a simple role are in turn simple. Also, since our tableau rules will not introduce new inclusion assertions, a role $r$ will be simple on a given branch $\Gamma$ if and only if $r$ is simple on the initial branch from which $\Gamma$ is obtained.

Our branches may now contain inclusion, reflexivity and transitivity assertions:

$$
s::=t x|r x y| x \doteq y|x \neq y| \perp\left|r \sqsubseteq r^{\prime}\right| R r \mid T r
$$


The modal expressions $t$ in formulas of the form $t x$ are restricted to contain no boxes $[r]_{n} s$ with $n>0$ unless $r$ is simple.

Following the ideas in [16, 18, 19, 17], we introduce the induced transition relation $\unrhd_{\Gamma}^{r}$ to reason about accessibility in the presence of inclusion axioms. Intuitively, $x \unrhd_{\Gamma}^{r} y$ means that in every model of $\Gamma, y$ is accessible from $x$ via $r$.

4.1. Extending Evidence. To account for the new types of formulas, we extend the evidence conditions as follows:

$$
\begin{aligned}
r \sqsubseteq r^{\prime} \in \Gamma & \Rightarrow \forall x, y \in \mathcal{N} \Gamma: r x y \in \tilde{\Gamma} \Rightarrow r^{\prime} x y \in \tilde{\Gamma} \\
R r \in \Gamma & \Rightarrow \forall x \in \mathcal{N} \Gamma: r x x \in \tilde{\Gamma} \\
T r \in \Gamma & \Rightarrow \forall x, y, z \in \mathcal{N} \Gamma: r x y \in \tilde{\Gamma} \wedge r y z \in \tilde{\Gamma} \Rightarrow r x z \in \tilde{\Gamma}
\end{aligned}
$$

It is easy to see that if $\Gamma$ satisfies the extended evidence conditions, the interpretation $\mathcal{I}$ constructed in the proof of Theorem 3.1 will satisfy the new formulas. Hence, Theorem 3.1 adapts to the extended system.

Theorem 4.1 (Model Existence). Every evident branch has a finite model.

4.2. Pre-evidence. To account for the new evidence conditions, one could imagine the following rules.

$$
\frac{r \sqsubseteq r^{\prime}, r x y}{r^{\prime} x y} \quad \frac{R r}{r x x} x \in \mathcal{N} \Gamma \quad \frac{T r, r x y, r y z}{r x z}
$$

In the presence of blocking, however, the rules are problematic. In particular, the rule for reflexivity renders the notion of quasi-evidence that we use for $\mathcal{T}$ ineffective to ensure termination. Once we add a reflexive edge $r x x$ to a branch $\Gamma, x$ will have an $r$-successor on $\Gamma$, meaning quasi-evidence will coincide with evidence for all $r$-diamonds on $x$. Similarly, the rule for transitivity is known to be incomplete in the presence of blocking [24].

We solve the problem by defining a weaker notion of evidence, called pre-evidence. To satisfy the pre-evidence conditions, we do not have to explicitly add reflexive or transitive edges during tableau construction. We will extend our tableau rules and the notion of quasi-evidence such that every open and maximal branch in the extended calculus can be completed to a pre-evident branch, which in turn can be made evident by adding the implicit edges.

We define the relation $\triangleright_{\Gamma}^{r}$ as the least relation such that:

$$
\begin{aligned}
& r x y \in \tilde{\Gamma} \Rightarrow x \triangleright_{\Gamma}^{r} y \\
& r^{\prime} \sqsubseteq r \in \Gamma, x \triangleright_{\Gamma}^{r^{\prime}} y \quad \Rightarrow \quad x \triangleright_{\Gamma}^{r} y
\end{aligned}
$$

The relation $\triangleright_{\Gamma}^{r}$ does not account for reflexivity. To do so, we extend it as follows:

$$
\unrhd_{\Gamma}^{r}:= \begin{cases}\triangleright_{\Gamma}^{r} \cup\left\{(x, y) \mid x, y \in \mathcal{N} \Gamma \wedge x \sim_{\Gamma} y\right\} & \text { if } \exists r^{\prime}: r^{\prime} \subseteq_{\Gamma}^{*} r \wedge R r^{\prime} \in \Gamma \\ \triangleright_{\Gamma}^{r} & \text { otherwise }\end{cases}
$$


The pre-evidence conditions are obtained from the evidence conditions by omitting the conditions for inclusion and reflexivity assertions and replacing the conditions for diamonds, boxes and transitivity assertions as follows:

$$
\begin{aligned}
\langle r\rangle_{n} t x \in \Gamma & \Rightarrow \exists^{n+1} Y: D_{\Gamma} Y \wedge \forall y \in Y: x \unrhd_{\Gamma}^{r} y \wedge t y \in \tilde{\Gamma} \\
{[r]_{n} t x \in \Gamma } & \Rightarrow\left|\left\{y \mid x \unrhd_{\Gamma}^{r} y, t y \notin \tilde{\Gamma}\right\} / \sim_{\Gamma}\right| \leq n \\
T r & \in \Gamma \Rightarrow \forall r^{\prime}, t, x, y:\left[r^{\prime}\right]_{0} t x \in \tilde{\Gamma} \wedge r \subseteq_{\Gamma}^{*} r^{\prime} \wedge x \triangleright_{\Gamma}^{r} y \Rightarrow[r]_{0} t y \in \tilde{\Gamma}
\end{aligned}
$$

Note that we do not need pre-evidence conditions for inclusion or reflexivity assertions as their semantics is taken care of by the way we define the relation $x \unrhd_{\Gamma}^{r} y$. Pre-evidence of individual formulas is defined analogously to the corresponding notion of evidence.

We now show that every pre-evident branch can be extended to an evident branch. Let the evidence closure $\hat{\Gamma}$ of a branch $\Gamma$ be defined as the least superset of $\Gamma$ such that:

$$
\begin{array}{r}
x \unrhd_{\Gamma}^{r} y \Rightarrow r x y \in \hat{\Gamma} \\
\operatorname{Tr} \in \Gamma \wedge r x y \in \hat{\Gamma} \wedge r y z \in \hat{\Gamma} \Rightarrow r x z \in \hat{\Gamma} \\
r \sqsubseteq r^{\prime} \in \Gamma \wedge r x y \in \hat{\Gamma} \Rightarrow r^{\prime} x y \in \hat{\Gamma}
\end{array}
$$

Note that, by construction, we have $r x y \in \hat{\tilde{\Gamma}} \Longleftrightarrow r x y \in \hat{\Gamma}$.

Lemma 4.2. Let $\Gamma$ be a branch and $r$ be simple on $\Gamma$. Then $x \unrhd_{\Gamma}^{r} y \Longleftrightarrow r x y \in \hat{\Gamma}$

Proof. Let $r$ be simple on $\Gamma$. The direction from left to right is immediate. The other direction can be shown by induction on the construction of $\hat{\Gamma}$ from $\Gamma$.

Lemma 4.3. Let $\Gamma$ be a branch and let $r x y \in \hat{\Gamma}$. Then either $x \unrhd_{\Gamma}^{r} y$, or there is some $r^{\prime}$ such that $\left\{r^{\prime} \sqsubseteq r, \operatorname{Tr}^{\prime}\right\} \subseteq \Gamma$ and

$$
\exists n \geq 2 \exists x_{1}, \ldots, x_{n}: x_{1}=x \wedge x_{n}=y \wedge \forall 1 \leq i<n: x_{i} \triangleright_{\Gamma}^{r^{\prime}} x_{i+1} .
$$

Proof. By induction on the construction of $\hat{\Gamma}$.

Theorem 4.4 (Evidence Completion). $\Gamma$ pre-evident $\Longrightarrow \hat{\Gamma}$ evident

Proof. It is easy to see that $\hat{\Gamma}$ satisfies the evidence conditions for inclusion, reflexivity and transitivity assertions. The only remaining evidence conditions that may be affected by adding edges to $\Gamma$ are the ones for diamonds and boxes. The rest of the evidence conditions is already satisfied by $\Gamma$ and hence also holds on $\hat{\Gamma}$.

The evidence condition for diamonds holds on $\hat{\Gamma}$ since the corresponding pre-evidence condition holds on $\Gamma$ and $x \unrhd_{\Gamma}^{r} y$ implies $r x y \in \hat{\Gamma}$ for all nominals $x, y$ and roles $r$.

It remains to show the evidence condition for boxes. Let $[r]_{n} s x \in \Gamma$ and $\mid\left\{y \mid x \unrhd_{\Gamma}^{r} y\right.$, $s y \notin \tilde{\Gamma}\} / \sim_{\Gamma} \mid \leq n$. It suffices to show: $\left|\{y \mid r x y \in \hat{\Gamma}, s y \notin \tilde{\Gamma}\} / \sim_{\Gamma}\right| \leq n$. We distinguish two cases. If $r$ is simple, the claim follows by Lemma 4.2, Otherwise, we must have $n=0$. Hence, it suffices to show that we have $s y \in \tilde{\Gamma}$ for every edge $r x y \in \hat{\Gamma}$. Let $r x y \in \hat{\Gamma}$. Then, by Lemma 4.3, two cases are possible. Either $x \unrhd_{\Gamma}^{r} y$, in which case the claim follows by the pre-evidence condition for boxes, or there is a transitive subrole $r^{\prime}$ of $r$ such that there are nominals $x_{1}, \ldots, x_{m}(m \geq 2)$ such that $x_{1}=x, x_{m}=y$ and $x_{i} \triangleright_{\Gamma}^{r^{\prime}} x_{i+1}$ for all $1 \leq i<n$. In this case, by induction on $m$ one can show that the pre-evidence condition for transitivity assertions applied to $r^{\prime}$ and $[r]_{n} s x \in \Gamma$ implies either $[r]_{n} s x_{m-1} \in \Gamma$ (true by assumption for $m=2$ ) or $\left[r^{\prime}\right]_{n} s x_{m-1} \in \tilde{\Gamma}$ (if $m>2$ ). Either way, the claim follows by the pre-evidence condition for boxes. 


$$
\begin{gathered}
\mathcal{R}_{\square} \frac{[r]_{n} t x}{\exists y, z \in Y, y \neq z: y \doteq z \mid \exists y \in Y: t y} Y \subseteq\left\{y \mid x \unrhd_{\Gamma}^{r} y\right\},|Y|=\left|Y / \sim_{\Gamma}\right|=n+1 \\
\mathcal{R}_{T} \frac{T r,\left[r^{\prime}\right]_{0} t x}{[r]_{0} t y} r \subseteq_{\Gamma}^{*} r^{\prime}, x \triangleright_{\Gamma}^{r} y
\end{gathered}
$$

Figure 4: New rules for $\mathcal{T}_{\sqsubseteq}$

4.3. Tableau Rules. The tableau rules for the extended calculus $\mathcal{T}_{\sqsubseteq}$ in Fig. 4 replace the original rule $\mathcal{R}_{\square}$ from Fig. 1 and add a new rule $\mathcal{R}_{T}$, which is necessary to achieve the pre-evidence condition for transitivity assertions. While the formulation of $\mathcal{R} \diamond$ remains unchanged, the rule will now have to use an adapted notion of quasi-evidence, which will be introduced in Sect. 4.4. For now, we assume $\mathcal{R}_{\diamond}$ is formulated with the restriction " $\langle r\rangle_{n} t x$ not pre-evident on $\Gamma$ " instead. Again, it is not hard to verify that the extended rules are sound.

4.4. Control. As it turns out, in the presence of role inclusion we have to modify the definition of patterns. It no longer suffices to consider patterns separately for each role. This is due to the fact that now, different roles may be constrained by inclusion assertions. Consider, for instance, the unsatisfiable branch

$$
\Gamma:=\left\{r \sqsubseteq r^{\prime},\langle r\rangle_{0} p x,\left\langle r^{\prime}\right\rangle_{0} \dot{\neg} p x,\left[r^{\prime}\right]_{1}(p \dot{\wedge} \dot{\neg} p) x, r^{\prime} x y, \dot{\neg} p y,\langle r\rangle_{0} p z, r z u, p u\right\}
$$

According to our previous notion of quasi-evidence, $\langle r\rangle_{0} p x$ is quasi-evident on $\Gamma$ as $x$ has no $r$-successor (even if we extend the set of successors to $\left\{y \mid x \triangleright_{\Gamma}^{r} y\right\}$ ) and $P_{\Gamma}^{r} x$ is expanded. Since the other two diamonds on $\Gamma$ are evident, $\Gamma$ is quasi-evident, witnessing the incompleteness of our previous definition of patterns.

Hence, we redefine the notion of a pattern as follows. Given a branch $\Gamma$, a pattern is a set of terms of the form $\mu s$, where $\mu \in\left\{\langle r\rangle_{n},[r]_{n} \mid r \in \operatorname{Rel} \Gamma, n \in \mathbb{N}\right\}$. We write $P_{\Gamma} x$ for the largest pattern $P$ such that $P \subseteq\{t \mid t x \in \tilde{\Gamma}\}$. We call $P_{\Gamma} x$ the pattern of $x$ on $\Gamma$. A pattern $P$ is expanded on $\Gamma$ if there are nominals $x, y$ and a role $r$ such that $x \triangleright_{\Gamma}^{r} y$ and $P \subseteq P_{\Gamma} x$. In this case, we say that $x$ expands $P$ on $\Gamma$. Note that here we use the relation $\triangleright_{\Gamma}^{r}$ rather than $\unrhd_{\Gamma}^{r}$. Otherwise, we would get the same problems with termination as outlined in Sect. 4.2,

A diamond formula $\langle r\rangle_{n} s x$ is quasi-evident on $\Gamma$ if it is either pre-evident on $\Gamma$ or $x$ has no successor on $\Gamma$ (i.e., there is no $y$ and $r$ such that $x \triangleright_{\Gamma}^{r} y$ ) and $P_{\Gamma} x$ is expanded on $\Gamma$. As before, we restrict the rule $\mathcal{R}_{\diamond}$ such that it can only be applied to diamond formulas that are not quasi-evident, and call a branch $\Gamma$ quasi-evident if it satisfies all of the pre-evidence conditions but the one for diamond formulas, which we again replace by

$$
\langle r\rangle_{n} t x \in \Gamma \Rightarrow\langle r\rangle_{n} t x \text { is quasi-evident on } \Gamma
$$

but now with the adapted notion of quasi-evidence.

Example 4.5. Figure 5 shows a tableau derivation in $\mathcal{T}_{\sqsubseteq}$ resulting in a quasi-evident branch. As in Example 3.5, we write $\Gamma_{n}$ for the branch up to line $n$. We observe:

- Since $r$ is reflexive and $r \sqsubseteq r^{\prime} \in \Gamma_{0}, r^{\prime}$ is also reflexive. Consequently, we have $x \unrhd_{\Gamma_{0}}^{r^{\prime}} x$, which explains why $\mathcal{R}_{\square}$ applies to $\left[r^{\prime}\right]_{0}\langle r\rangle_{0} p x \in \Gamma_{0}$. 
0. $r \sqsubseteq r^{\prime}, R r, T r^{\prime},\left[r^{\prime}\right]_{0}\langle r\rangle_{0} p x,\left\langle r^{\prime}\right\rangle_{0} q x$

$\begin{array}{lcc}\text { 1. } & \langle r\rangle_{0} p x & \mathcal{R}_{\square} \\ \text { 2. } & r x y, p y & \mathcal{R}_{\diamond} \\ \text { 3. } & r^{\prime} x z, q z & \mathcal{R}_{\diamond} \\ 4 . & {\left[r^{\prime}\right]_{0}\langle r\rangle_{0} p z} & \mathcal{R}_{T} \\ 5 . & \langle r\rangle_{0} p z & \mathcal{R}_{\square} \\ 6 . & r z u, p u & \mathcal{R}_{\diamond}\end{array}$

Figure 5: Tableau derivation for $\left\{r \sqsubseteq r^{\prime}, R r, T r^{\prime},\left[r^{\prime}\right]_{0}\langle r\rangle_{0} p x,\left\langle r^{\prime}\right\rangle_{0} q x\right\}$

- The rule $\mathcal{R}_{T}$ propagates $\left[r^{\prime}\right]_{0}\langle r\rangle_{0} p$ to $z$ but not to $y$ since $r$ is not (necessarily) transitive.

- In $\mathcal{T},\langle r\rangle_{0} p z \in \Gamma_{5}$ would be quasi-evident since $P_{\Gamma_{5}}^{r} x=P_{\Gamma_{5}}^{r} z$. In $\mathcal{T}_{\sqsubseteq}$, however, $\mathcal{R}_{\diamond}$ applies to $\langle r\rangle_{0} p z$ since $P_{\Gamma_{5}} x=\left\{\langle r\rangle_{0} p,\left\langle r^{\prime}\right\rangle_{0} q,\left[r^{\prime}\right]_{0}\langle r\rangle_{0} p\right\} \neq\left\{\langle r\rangle_{0} p,\left[r^{\prime}\right]_{0}\langle r\rangle_{0} p\right\}=P_{\Gamma_{5}} z$.

Lemma 4.6. Let $\Gamma, \Delta$ be branches such that $\left\{r \sqsubseteq r^{\prime} \mid r \sqsubseteq r^{\prime} \in \Gamma\right\}=\left\{r \sqsubseteq r^{\prime} \mid r \sqsubseteq r^{\prime} \in \Delta\right\}$. Let $x, y, u, v$ be nominals such that $\{r \mid r x y \in \tilde{\Gamma}\}=\{r \mid r u v \in \tilde{\Delta}\}$. Then, for all $r$, $x \triangleright_{\Gamma}^{r} y \Leftrightarrow u \triangleright_{\Delta}^{r} v$.

Proof. Let $\Gamma, \Delta, x, y, u$ and $v$ be as required. Let $r$ be a role. We show $x \triangleright_{\Gamma}^{r} y \Rightarrow u \triangleright_{\Delta}^{r} v$ by induction on the derivation of $x \triangleright_{\Gamma}^{r} y$. The other direction follows analogously by induction on the derivation of $u \triangleright_{\Delta}^{r} v$. Assume $x \triangleright_{\Gamma}^{r} y$. We distinguish two cases:

- $r x y \in \tilde{\Gamma}$. Then, by assumption, $r u v \in \tilde{\Delta}$, and so $u \triangleright_{\Delta}^{r} v$.

- There is some $r^{\prime}$ such that $r^{\prime} \sqsubseteq r \in \Gamma$ and $x \triangleright_{\Gamma}^{r^{\prime}} y$. By the inductive hypothesis, we have $u \triangleright_{\Delta}^{r^{\prime}} v$. Moreover, by assumption, $r^{\prime} \sqsubseteq r \in \Delta$. Hence, $u \triangleright_{\Delta}^{r} v$.

Lemma 4.7. Let $\Gamma$ be a quasi-evident branch and let $\langle r\rangle_{n} s x$ be not pre-evident on $\Gamma$. Let $y$ expand $P_{\Gamma} x$ on $\Gamma$ and let $\Delta:=\Gamma \cup\left\{r^{\prime} x z \mid r^{\prime} y z \in \tilde{\Gamma}\right\}$. Then:

(1) $\forall r^{\prime}, z: x \triangleright_{\Delta}^{r^{\prime}} z \Longleftrightarrow y \triangleright_{\Gamma}^{r^{\prime}} z$ and $x \unrhd_{\Delta}^{r^{\prime}} z \Longleftrightarrow y \unrhd_{\Gamma}^{r^{\prime}} z$,

(2) $\forall r^{\prime}, m, t:\left\langle r^{\prime}\right\rangle_{m} t \in P_{\Gamma} x \Longrightarrow\left\langle r^{\prime}\right\rangle_{m} t x$ pre-evident on $\Delta$,

(3) $\langle r\rangle_{n} s x$ pre-evident on $\Delta$,

(4) $\forall r^{\prime}, m, t, z:\left\langle r^{\prime}\right\rangle_{m} t z$ pre-evident on $\Gamma \Longrightarrow\left\langle r^{\prime}\right\rangle_{m} t z$ pre-evident on $\Delta$,

(5) $\Delta$ quasi-evident.

Proof. We begin with (1). Let $r^{\prime}$ be a role and $z$ a nominal. We will only show the first equivalence since the other claim easily follows. Since $\langle r\rangle_{n} s x$ is quasi-evident but not evident on $\Gamma, x$ has no successor on $\Gamma$. Hence, by construction, $\left\{r^{\prime} \mid r^{\prime} x z \in \tilde{\Delta}\right\}=\left\{r^{\prime} \mid r^{\prime} y z \in \tilde{\Gamma}\right\}$. The claim follows by Lemma 4.6.

Claims (2-4) are shown analogously to the corresponding claims of Lemma 3.6.

Now to (5). The only conditions that might in principle be violated in $\Delta$ are the quasi-evidence condition for diamonds of the form $\left\langle r^{\prime}\right\rangle_{m} t z \in \Delta$ where $z \sim_{\Delta} x$, the evidence condition for boxes $\left[r^{\prime}\right]_{m} t z \in \Delta$ where $z \sim_{\Delta} x$, and the evidence condition for transitivity assertions $\operatorname{Tr}^{\prime} \in \Delta$.

For diamonds of the above form, the quasi-evidence condition holds by (2).

For transitivity assertions, it suffices to show that for every $r_{1}, r_{2}$ such that $\operatorname{Tr}_{1} \in \Gamma$, $r_{1} \subseteq_{\Gamma}^{*} r_{2}$, and $\left[r_{2}\right]_{0} t x \in \tilde{\Gamma}$, and for all $z$ such that $x \triangleright_{\Delta}^{r_{1}} z$, it holds $\left[r_{1}\right]_{0} t z \in \tilde{\Gamma}$. Since 
$P_{\Gamma} y \supseteq P_{\Gamma} x$, we have $\left[r_{2}\right]_{0} t y \in \tilde{\Gamma}$. The claim now follows by (1) and the quasi-evidence condition for $\operatorname{Tr}_{1} \in \Gamma$.

The claim for boxes follows analogously (we exploit $P_{\Gamma} y \supseteq P_{\Gamma} x$ and (1)).

Theorem 4.8 (Pre-evidence Completion). For every quasi-evident branch $\Gamma$ there is a pre-evident branch $\Delta$ such that $\Gamma \subseteq \Delta$.

Proof. Proceeds analogously to the proof of Theorem 3.7 with Lemma 4.7 in place of Lemma 3.6.

Theorem 4.9 (Quasi-evidence). Every open and maximal branch in $\mathcal{T}_{\sqsubseteq}$ is quasi-evident.

Proof. Proceeds analogously to the proof of Theorem 3.8. The additional case for transitivity assertions is straightforward.

4.5. Termination. The termination proof for $\mathcal{T}_{\sqsubseteq}$ proceeds analogously to the proof for $\mathcal{T}$. Let us sketch what needs to be adapted. Because of the rule $\mathcal{R}_{T}$, the set $\mathcal{S} \Gamma$ of modal expressions occurring on $\Gamma$ needs to be extended as follows: $\mathcal{S}^{\prime} \Gamma:=\mathcal{S} \Gamma \cup\left\{[r]_{0} s \mid r \subseteq_{\Gamma}^{*} r^{\prime} \wedge\right.$ $\left.\left[r^{\prime}\right]_{0} s \in \mathcal{S} \Gamma\right\}$. With the extended definition of $\mathcal{S}$, Proposition 3.9 holds for $\mathcal{T}_{\sqsubseteq}$. Lemma 3.10 is modified as follows:

Lemma 4.10. Let $s$ be of the form $\langle r\rangle_{n} t x$ or $E_{n} t x$. If $s$ is (pre-)evident on $\Gamma$ and $\Gamma \subseteq \Delta$, then $s$ is (pre-)evident on $\Delta$.

Proposition 3.11 is unaffected by the extensions to the calculus. Proposition 3.12 is adapted as follows:

Proposition 4.11. If $\mathcal{R}_{\diamond}$ is applicable to a formula $\langle r\rangle_{n} s x \in \Gamma$, then either

(1) $x$ has a successor on $\Gamma$, or

(2) $P_{\Gamma} x$ is not expanded on $\Gamma$.

Also, analogously to Lemma 3.13, the expandedness of our extended patterns is preserved by tableau rule application. Lemma 3.14 and Proposition 3.15 remain valid if we redefine

$$
\psi_{\diamond}^{X} \Gamma:=\mid\left\{\langle r\rangle_{n} s \in \mathcal{S}^{\prime} \Gamma \mid \exists x \in X:\langle r\rangle_{n} s x \text { not pre-evident on } \Gamma\right\} \mid
$$

and $\psi_{\diamond} \Gamma$ accordingly, with the modified definition of a successor.

\section{Conclusion}

We have presented a terminating tableau calculus for graded hybrid logic with global modalities and role hierarchies. Following [8, 7, 24, our calculus is cumulative, representing state equality abstractly via an equivalence relation (declarative approach). The existing calculi for equivalent and stronger logics [18, 19, 17] work on possibly cyclic graph structures and treat equality by destructive graph transformation during tableau construction (procedural approach). The procedural approach encompasses algorithmic decisions that are not present in the more abstract declarative approach. From a declarative calculus we can always obtain a procedural system by refinement.

Exploiting an extended pattern-based blocking technique and the cumulativity of our calculus, we have proved a NExPTIME complexity bound for the associated decision procedure. To ensure termination of pattern-based blocking in the presence of reflexivity, we 
differentiated between the induced transition relation $\unrhd_{\Gamma}^{r}$ and its non-reflexive counterpart $\triangleright_{\Gamma}^{r}$. The implementation of pattern-based blocking for a hybrid language with global modalities [15] reveals its considerable practical potential. We consider it a promising project to implement the extended version of pattern-based blocking presented in this paper and compare its performance to that of established blocking techniques.

Following related work [16, 20, 18, 19, 17, we restrict the language decided by our calculus to contain no graded boxes on complex roles. As shown by Horrocks, Sattler and Tobies [20, this restriction is essential for decidability of logics extending $\mathcal{S H \mathcal { I N }}$. In the absence of inverse roles $(\mathcal{I})$, however, the restriction of graded boxes to simple roles can be significantly relaxed [26]. In [25, we give a terminating tableau calculus for $\mathcal{S O} \mathcal{Q}$ extended by graded boxes on transitive roles. The logic extends the decidable fragment of [26] by nominals but lacks inclusion assertions that are allowed (with some restrictions) in [26]. It remains an open problem to design an efficient tableau calculus for the full decidable fragment of [26]. Also, it is still open if the fragment of [26] remains decidable when extended by nominals.

Acknowledgement. We would like to thank our referees for their valuable comments that helped to improve the paper.

\section{REFERENCES}

[1] Carlos Areces and Balder ten Cate. Hybrid logics. In Blackburn et al. 6], pages 821-868.

[2] Franz Baader, Martin Buchheit, and Bernhard Hollunder. Cardinality restrictions on concepts. Artif. Intell., 88(1-2):195-213, 1996.

[3] Franz Baader, Diego Calvanese, Deborah L. McGuinness, Daniele Nardi, and Peter F. Patel-Schneider, editors. The Description Logic Handbook: Theory, Implementation and Applications. Cambridge University Press, 2nd edition, 2007.

[4] Franz Baader and Carsten Lutz. Description logic. In Blackburn et al. 6], pages 757-820.

[5] Franz Baader and Ulrike Sattler. An overview of tableau algorithms for description logics. Stud. Log., 69(1):5-40, 2001.

[6] Patrick Blackburn, Johan van Benthem, and Frank Wolter, editors. Handbook of Modal Logic, volume 3 of Studies in Logic and Practical Reasoning. Elsevier, 2007.

[7] Thomas Bolander and Patrick Blackburn. Termination for hybrid tableaus. J. Log. Comput., 17(3):517554, 2007.

[8] Thomas Bolander and Torben Braüner. Tableau-based decision procedures for hybrid logic. J. Log. Comput., 16(6):737-763, 2006.

[9] Chad E. Brown and Gert Smolka. Analytic tableaux for simple type theory and its first-order fragment. Log. Methods Comput. Sci., 6(2), 2010.

[10] William M. Farmer. The seven virtues of simple type theory. J. Appl. Log., 6(3):267-286, 2008.

[11] Maurizio Fattorosi-Barnaba and Francesco De Caro. Graded modalities I. Stud. Log., 44(2):197-221, 1985.

[12] Kit Fine. In so many possible worlds. Notre Dame J. Form. Log., 13(4):516-520, 1972.

[13] Valentin Goranko and Solomon Passy. Using the universal modality: Gains and questions. J. Log. Comput., 2(1):5-30, 1992.

[14] Daniel Götzmann. Spartacus: A Tableau Prover for Hybrid Logic. M.Sc. thesis, Saarland University, 2009.

[15] Daniel Götzmann, Mark Kaminski, and Gert Smolka. Spartacus: A tableau prover for hybrid logic. In Thomas Bolander and Torben Braüner, editors, Proc. 6th Workshop on Methods for Modalities (M4M-6), volume 262 of Electr. Notes Theor. Comput. Sci., pages 127-139. Elsevier, 2010.

[16] Ian Horrocks. Optimising Tableaux Decision Procedures for Description Logics. PhD thesis, University of Manchester, 1997. 
[17] Ian Horrocks, Oliver Kutz, and Ulrike Sattler. The even more irresistible $\mathcal{S} \mathcal{R O} \mathcal{I} \mathcal{Q}$. In Patrick Doherty, John Mylopoulos, and Christopher A. Welty, editors, Proc. 10th Intl. Conf. on Principles of Knowledge Representation and Reasoning (KR 2006), pages 57-67. AAAI Press, 2006.

[18] Ian Horrocks and Ulrike Sattler. Ontology reasoning in the $\mathcal{S H O Q}(\mathrm{D})$ description logic. In Bernhard Nebel, editor, Proc. 17th Intl. Joint Conf. on Artificial Intelligence (IJCAI 2001), pages 199-204. Morgan Kaufmann, 2001.

[19] Ian Horrocks and Ulrike Sattler. A tableau decision procedure for $\mathcal{S H O I Q}$. J. Autom. Reasoning, 39(3):249-276, 2007.

[20] Ian Horrocks, Ulrike Sattler, and Stephan Tobies. Practical reasoning for very expressive description logics. L. J. IGPL, 8(3):239-263, 2000.

[21] Mark Kaminski, Sigurd Schneider, and Gert Smolka. Terminating tableaux for graded hybrid logic with global modalities and role hierarchies. In Martin Giese and Arild Waaler, editors, TABLEAUX 2009, volume 5607 of $L N C S$ (LNAI), pages 235-249. Springer, 2009.

[22] Mark Kaminski and Gert Smolka. Terminating tableaux for hybrid logic with the difference modality and converse. In Alessandro Armando, Peter Baumgartner, and Gilles Dowek, editors, IJCAR 2008, volume 5195 of $L N C S$ (LNAI), pages 210-225. Springer, 2008.

[23] Mark Kaminski and Gert Smolka. Hybrid tableaux for the difference modality. In Carlos Areces and Stéphane Demri, editors, Proc. 5th Workshop on Methods for Modalities (M4M-5), volume 231 of Electr. Notes Theor. Comput. Sci., pages 241-257. Elsevier, 2009.

[24] Mark Kaminski and Gert Smolka. Terminating tableau systems for hybrid logic with difference and converse. J. Log. Lang. Inf., 18(4):437-464, 2009.

[25] Mark Kaminski and Gert Smolka. Terminating tableaux for $\mathcal{S O} \mathcal{Q}$ with number restrictions on transitive roles. In Cristian S. Calude and Vladimiro Sassone, editors, TCS 2010, volume 323 of IFIP AICT, pages 213-228. Springer, 2010.

[26] Yevgeny Kazakov, Ulrike Sattler, and Evgeny Zolin. How many legs do I have? Non-simple roles in number restrictions revisited. In Nachum Dershowitz and Andrei Voronkov, editors, LPAR 200\%, volume 4790 of LNCS (LNAI), pages 303-317. Springer, 2007.

[27] Saul A. Kripke. Semantical analysis of modal logic I: Normal modal propositional calculi. Z. Math. Logik Grundlagen Math., 9:67-96, 1963.

[28] Boris Motik, Rob Shearer, and Ian Horrocks. Optimized reasoning in description logics using hypertableaux. In Frank Pfenning, editor, CADE-21, volume 4603 of LNCS (LNAI), pages 67-83. Springer, 2007.

[29] Hans Jürgen Ohlbach, Renate A. Schmidt, and Ullrich Hustadt. Translating graded modalities into predicate logic. In Heinrich Wansing, editor, Proof Theory of Modal Logic, volume 2 of Applied Logic Series, pages 253-291. Kluwer, 1996.

[30] Ulrike Sattler. A concept language extended with different kinds of transitive roles. In Günther Görz and Steffen Hölldobler, editors, KI-96, volume 1137 of LNCS (LNAI), pages 333-345. Springer, 1996.

[31] Wiebe van der Hoek and Maarten de Rijke. Counting objects. J. Log. Comput., 5(3):325-345, 1995.

This work is licensed under the Creative Commons Attribution-NoDerivs License. To view a copy of this license, visit http://creativecommons.org/licenses/by-nd/2.0/ or send a letter to Creative Commons, 171 Second St, Suite 300, San Francisco, CA 94105, USA, or Eisenacher Strasse 2, 10777 Berlin, Germany 